\title{
Flame Resistant Nylon-6,6 Composites with Improved Mechanical Strength by the Combination of Additive- and Reactive-Type Flame Retardants
}

\author{
Toshiyuki Kanno, ${ }^{1, \dagger}$ Hironori Yanase, ${ }^{1}$ Yoshinobu Sugata, ${ }^{1}$ and Kiyotaka SHIGeHARA ${ }^{2}$ \\ ${ }^{1}$ Production Technol. Lab., Fuji Electric Advanced Technology Co., Ltd., 1 Fuji-machi, Hino 191-8502, Japan \\ ${ }^{2}$ Strategic Research Initiative for Future Nano-science and Technology, Institute of Symbiotic Science and Technology, \\ Tokyo University of Agriculture and Technology, 2-24-16 Nakacho, Koganei 184-8588, Japan
}

(Received November 17, 2006; Accepted January 12, 2007; Published February 26, 2007)

\begin{abstract}
Characteristics of flame-resistant nylon-6,6 (PA66) composites with improved mechanical strength at high temperatures were studied. The composites prepared by mixing PA66 with organic phosphorus flame retardants of additive-type and reactive-type, the latter of which carrying allyl functionalities turned the resulting composite infusible after the $\gamma$-ray irradiation cross-linking. The storage modulus became constant after increased with the exposure period (at [flame retardant] $=$ const.) or the amount of the reactive flame retardant (at exposure period $=$ const.). The cross-linked composites not only showed rubber like elasticity even at temperatures higher than the melting point of uncross-linked PA66, but also provided no drip upon combustion. Although the cross-linked composites with the reactive-type flame retardant gave insufficient flame-resistant grade, the one with both additive- and reactive-type flame retardants realized the UL94/V-0 grade with satisfactory mechanical strength.

[doi:10.1295/polymj.PJ2006164]

KEY WORDS Flame Retardant / Nylon-6,6 / Cross-Linking / UL94 /
\end{abstract}

Organic polyhalogenated molecules, such as polybrominated biphenyls or related compounds, inorganic phosphorous red, antimony oxide and so on have been utilized as typical additives or fillers to give flame retardation properties to polymer materials. However, as the environmental safety has become increasingly significant in recent years, these materials should be gradually replaced or even avoided due to the toxicity in use or after combustion. Instead, the metal hydroxides such as $\mathrm{Al}(\mathrm{OH})_{3}$ or $\mathrm{Mg}(\mathrm{OH})_{2}$, the metal phosphorus salt such as aluminum phosphate, organic phosphorous compounds and nitrogenous substances have been used as the flame retardants which are considered to be environmentally safe, but they need to be added massively to base resins to satisfy the desired flame-resistant grade in use, that often causes the following drawbacks: ${ }^{1}$

Polymer alloy-type flame retardants (organic phosphorous compounds, etc.)

(a) bleed-out of flame retardant on the surface of molded products.

(b) decrease of mechanical strength and/or heatresistant properties due to plasticization.

Disperse-type flame retardants (metal hydroxides fine grain, etc.)

(c) decrease of mechanical strength due to the grain boundary problem.

(d) decrease of electric resistance due to hydroxide groups. (e) decrease of humidity resistance due to hydroxide groups.

As for the polymer alloy systems, the problems (a) and (b) are considered to be minimized by cross-linking, of which strategy is classified into 4 categories. Hereafter AFR, RFR and CR denote respectively the "Additive-type" flame retardants, "Reactive-type" flame retardants carrying cross-linkable functionalities and cross-linking reagent.

(1) AFR + thermosetting resin

(2) $\mathrm{AFR}+\mathrm{CR}+$ thermoplastic resin

(3) RFR + thermoplastic resin

(4) $\mathrm{AFR}+\mathrm{RFR}+$ thermoplastic resin

Thick solutions of unsaturated polyesters dissolved in vinyl monomers are often used as thermosetting resins. However the improvement of "bleed-out" will not be sufficient in the case (1), since the network formation of thermosetting resins usually cause phase separation during the curing period. In order to maintain the advantage to use thermoplastic resins such as easier shaping, for the cases (2)-(4) the cross-linking reaction must not occur during the extrusion or molding, but is allowed to advance after the completion of shaping. For this purpose, the $\gamma$-ray irradiation postcrosslinking via allyl functionalities that can proceed even at r.t. is superior to other methods. Unfortunately the commercially available polyallyl type CRs such as triallyl isocyanurate (TAIC) or related compounds vaporize at the temperature where the common thermo-

${ }^{\dagger}$ To whom correspondence should be addressed (Tel: +81-42-586-1071, Fax: +81-42-582-3663, E-mail: kanno-toshiyuki@fujielectric.co.jp). 
plastic resins such as PA66 (nylon-6,6) become fused enough for the extrusion and molding processing. If new CRs are designed to have higher molecular weight in order to endure the elevated temperature, it is better to introduce phosphorus and nitrogen atoms in the nuclei, i.e., RFRs or the polyallyl-functionalized flame retardants. We developed such RFRs, and confirmed that the mechanical strength and heat-resistant properties of the RFR/PA66 composites were satisfactorily improved by the $\gamma$-ray irradiation cross-linking. ${ }^{2}$ If the durability of thermoplastic resins with enough flame retardation becomes comparable to those of thermosetting resins by cross-linking the thermoplastic resins with RFR, then no thermosetting resin has to be used from the beginning, which means that the thermoplastic resin can supersede the thermosetting resin and which presents the following advantages: (i) about a third to half of resin usually to be purged becomes recyclable, (ii) there is the possibility of recycling the final products by decomposing the cross-linked portion by means of oxidization or heating, and (iii) post-crosslinked thermoplastic resins are lighter and cheaper than thermosetting resins. However, such cross-linked RFR composites sometimes gave unsatisfactory flame retardation, possibly because the difficulty arouse in the evolution of phosphorus-containing fragments to the burning surface due to the covalent anchoring of flame retardant nuclei. If this is true, the addition of AFR besides RFR to the extent that does not cause bleed-out and plasticization maybe effective. The case (4) corresponding to this strategy was not yet examined due to the difficulty in finding suitable AFR and RFR combination, as the typical disperse-type flame retardants such as metal hydroxides usually cause serious deterioration in electric properties of the resulting composites, i.e., (d) and (e) described above.

In order to avoid the problems (d) and (e) of the disperse-type composites, instead of metal hydroxide flame retardants, melamine-polyphosphate complexes (MP complex $)^{3,4}$ and aluminum organic phosphinate salts with no hydroxide are promising candidates as AFR. ${ }^{5,6}$ When such disperse-type AFRs are chosen, especially the case (4) as well as the case (2) can be hopefully examined without causing the problems (d) and (e), satisfying both the enough mechanical strength and flame retardation.

The MP complex, the phosphinate salt and the mixture of them were known to exhibit excellent flame retardation effect against PA66 or related resins. ${ }^{7}$ However, according to our experience, the MP complex and the mixture partially degraded during the kneading and extruding processes of PA66 composites at about $260{ }^{\circ} \mathrm{C}$ to form impurities that often adhered tenaciously to molding dies to enforce frequent and unavoidable cleaning after every 20 to 50 shots. Therefore, in the present paper, we chose the phosphinate salt as AFR that does not degrade below $400{ }^{\circ} \mathrm{C}$. The effects of AFR/RFR dual flame retardants mixed in PA66 resin were examined before and after the $\gamma$-ray cross-linking in respect to not only the grade of flame retardation estimated by the UL94/V burning tests and the surface analysis of burnt samples but also the mechanical strength of the resulting composites evaluated by dynamic viscoelasticity measurements.

\section{EXPERIMENTAL}

\section{Materials and Samples}

Base Resin, CR, AFR and Fillers The reagents and the following materials were used as received.

- Base resin: PA66 (polyamide-66 = nylon-6,6, Ube Industries; $2020 \mathrm{~B}, M_{\mathrm{n}}=20,000, M_{\mathrm{w}} / M_{\mathrm{n}}=$ 1.8-2.0)

- AFR: Aluminum tris(diethylphosphinate) (Clariant; Exolit OP-1230, median diameter $=10.1$ $\mu \mathrm{m})$

- Reinforcements: Glass fiber (Asahi Fiber-Glass; 03JAFT-2A) with $10 \mu \mathrm{m}$ dia. $\times 3 \mathrm{~mm} l$.

- Inorganic fillers: $\mathrm{SiO}_{2}$ powder (Fuji Silysia Chemical; Sylisia 530, hydrophilic surface and an average particle diameter of $2.7 \mu \mathrm{m}$ ), Talc (Hayashi Kasei, MICRON WHITE \#5000S, an average particle diameter of $2.8 \mu \mathrm{m})$

Synthesis of RFR 4, $4^{\prime}$-bis $\left(N, N, N^{\prime}, N^{\prime}\right.$-tetraallyldiaminophosphoryl)biphenyl, an organic phosphorous compound with octaallyl functionality, of which molecular structure is shown in Scheme 1, was utilized as an RFR. 4,4'-Biphenol $(18.7 \mathrm{~g}, 0.100 \mathrm{~mol})$ in THF $(150 \mathrm{~mL})$ was added dropwise into the mixture of phosphoryl chloride $(122.7 \mathrm{~g}, 0.800 \mathrm{~mol})$ and triethylamine (TEA; $22.26 \mathrm{~g}, 0.220 \mathrm{~mol}$ ) as $\mathrm{HCl}$ remover in THF $(150 \mathrm{~mL})$ and reacted at $60^{\circ} \mathrm{C}$ for $12 \mathrm{~h}$. The resulting 4,4'-bis(dichloro-phosphoryl)biphenyl (DCPB) obtained by filtration and evaporation to exclude amine salt and excess reagents or solvent, respectively, was dissolved in THF $(200 \mathrm{~mL})$ and added dropwise to the mixture of diallylamine $(69.66 \mathrm{~g}, 0.800$ $\mathrm{mol})$ and TEA $(44.52 \mathrm{~g}, 0.440 \mathrm{~mol})$ in $150 \mathrm{~mL}$ of THF. The mixture was reacted at $60^{\circ} \mathrm{C}$ for $18 \mathrm{~h}$, filtered, evaporated, and re-dissolved in $\mathrm{CHCl}_{3}$. The so-

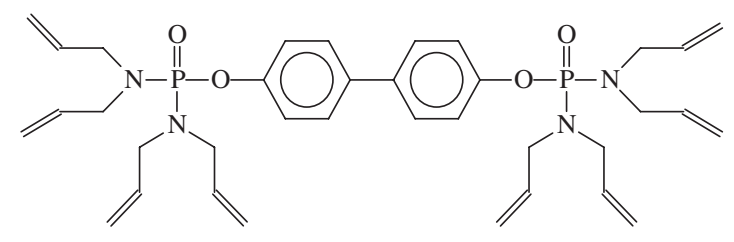

Scheme 1. 
Flame Resistant Nylon-6,6 Composites with Improved Mechanical Strength

Table I. Composite composition and results of UL94V test

\begin{tabular}{|c|c|c|c|c|c|c|c|c|}
\hline \multirow[b]{2}{*}{ Composite } & \multicolumn{4}{|c|}{ Composition (wt/wt \%) ${ }^{\mathrm{a}}$} & \multicolumn{4}{|c|}{ Results of flaming test } \\
\hline & AFR & $\mathrm{CR}^{\mathrm{b}}$ & RFR & PA66 & $\begin{array}{c}\gamma \text {-ray dose } \\
(\mathrm{kGy})\end{array}$ & $\begin{array}{l}\text { Char yield }^{\mathrm{c}} \\
(\mathrm{wt} / \mathrm{wt} \%)\end{array}$ & $\begin{array}{l}\text { UL94/V } \\
\text { grade }\end{array}$ & Drip \\
\hline \multirow{2}{*}{ Control } & \multirow{2}{*}{0} & \multirow{2}{*}{0} & \multirow{2}{*}{0} & \multirow{2}{*}{54} & 0 & $<0.1$ & NG & Yes \\
\hline & & & & & 40 & $<0.1$ & NG & Yes \\
\hline \multirow{3}{*}{$I$} & \multirow{3}{*}{12} & \multirow{3}{*}{2} & \multirow{3}{*}{0} & \multirow{3}{*}{40} & 0 & 12 & V-1 & No \\
\hline & & & & & 25 & 13 & $\mathrm{~V}-1$ & No \\
\hline & & & & & 40 & 13 & $\mathrm{~V}-1$ & No \\
\hline \multirow{3}{*}{ II } & \multirow{3}{*}{0} & \multirow{3}{*}{0} & \multirow{3}{*}{12} & \multirow{3}{*}{42} & 0 & 6 & NG & No \\
\hline & & & & & 25 & 7 & NG & No \\
\hline & & & & & 40 & 6 & NG & No \\
\hline \multirow{3}{*}{ III } & \multirow{3}{*}{12} & \multirow{3}{*}{0} & \multirow{3}{*}{8} & \multirow{3}{*}{34} & 0 & 12 & $\mathrm{~V}-1$ & No \\
\hline & & & & & 25 & 12 & $\mathrm{~V}-1$ & No \\
\hline & & & & & 40 & 11 & $\mathrm{~V}-1$ & No \\
\hline \multirow{3}{*}{$I V$} & \multirow{3}{*}{12} & \multirow{3}{*}{0} & \multirow{3}{*}{4} & \multirow{3}{*}{38} & 0 & 10 & $\mathrm{~V}-1$ & No \\
\hline & & & & & 25 & 10 & V-1 & No \\
\hline & & & & & 40 & 10 & $\mathrm{~V}-1$ & No \\
\hline \multirow{3}{*}{$\boldsymbol{V}$} & \multirow{3}{*}{12} & \multirow{3}{*}{0} & \multirow{3}{*}{2} & \multirow{3}{*}{40} & 0 & 11 & $\mathrm{~V}-0$ & No \\
\hline & & & & & 25 & 11 & $\mathrm{~V}-0$ & No \\
\hline & & & & & 40 & 12 & V-0 & No \\
\hline
\end{tabular}

${ }^{\mathrm{a}}$ The contents of GF, Talc and $\mathrm{SiO}_{2}$ powder were kept content as 31,4 and $11 \mathrm{wt} / \mathrm{wt} \%$, resp. ${ }^{\mathrm{b}}$ Crosslinking reagent $=$ triallyl isocyanurate. ${ }^{\mathrm{c}} \mathrm{Char}$ yield $=$ percentage of remaining organics at $600{ }^{\circ} \mathrm{C}$. Also see text and Figure 7.

lution was rinsed 3-times with water, evaporated and dried in vacuo at $40^{\circ} \mathrm{C}$ to give the desired product as pale yellow waxy solid typically in $92-95 \%$ yield. NMR $\left(\delta\right.$ ppm, $\left.\mathrm{CDCl}_{3}\right): \mathrm{CH}_{2}=5.1,=\mathrm{CH}-5.6,-\mathrm{CH}_{2}-$ 3.5, Ph-H 7.6, 7.2, and TOF-Mass $(m / z)=664,665$ (calcd. 662.7). The purity checked by HPLC (biphenyl absorption band as index) was about $97 \%$.

Test Samples The base resin PA66, AFR and/or RFR, reinforcements and fillers were mixed in a 2axis extruder TEX30 $\alpha$ (Japan Steel Works) at $280^{\circ} \mathrm{C}$ under $\mathrm{N}_{2}$ atmosphere to produce pelletized stocks, of which compositions were summarized in Table I. When TAIC was used as CR, the kneading and extruding processes were carried out in the closed systems in order to avoid the escape of TAIC by vaporization. The injection molding machine ROBOSHOT $\alpha-50 \mathrm{C}$ (FANUC) was employed to prepare the test pieces from the pellets. The dimensions of the samples for the temperature-dependent dynamic viscoelasticity measurement were $40 \times 10 \times 0.95 t \mathrm{~mm}$, and those for the UL94/V flammability test were $127 \times$ $12.7 \times 0.8 \mathrm{tmm}$.

\section{Cross-Linking Process}

The test pieces were exposed to 25 or $40 \mathrm{kGy} \gamma$-ray from the radiation source of ${ }^{60} \mathrm{Co}$ (Japan Irradiation Service) at r.t. and under nitrogen atmosphere.

\section{Dynamic Viscoelasticity Measurement}

The temperature-dependence of dynamic viscoelas- ticity was measured by a Paar PHYSICA UDS200 viscoelastometer under the following test conditions: applied distortion $=0.2 \%$, driving frequency $=1 \mathrm{~Hz}$, temperature range $=45-320^{\circ} \mathrm{C}$ at $5^{\circ} \mathrm{C} / \mathrm{min}$ increment.

\section{Flame Retardation Measurement}

The flammability test was conducted according to the UL94/V (vertical burning) flammability test (ASTM D3801). After burning the test blade for $10 \mathrm{~s}$ with the methane/air Bunsen burner and the burner was removed, the period required for the spontaneous extinguishment was measured. After the extinguishment the burnt sample was conducted to 2 nd run measurement of the period, repeating the same procedure described above. The inflammability grade was classified according to the burning period of 1 st and 2nd run ( $=\mathrm{t}_{1}$ and $\mathrm{t}_{2}$, resp.) into UL94/V-0 $\left(\mathrm{t}_{1}\right.$ or $\mathrm{t}_{2}<$ $10 \mathrm{~s}, \mathrm{t}_{1}+\mathrm{t}_{2}<30 \mathrm{~s}$, no drip), V-1 ( $\mathrm{t}_{1}$ or $\mathrm{t}_{2} \leqq 30 \mathrm{~s}$, $\mathrm{t}_{1}+\mathrm{t}_{2} \leqq 60 \mathrm{~s}$, no drip), $\mathrm{V}-2$ ( $\mathrm{t}_{1}$ or $\mathrm{t}_{2} \leqq 30 \mathrm{~s}, \mathrm{t}_{1}+\mathrm{t}_{2} \leqq$ $60 \mathrm{~s}$, drip), and NG $\left(\mathrm{t}_{1}\right.$ or $\mathrm{t}_{2}>30 \mathrm{~s}, \mathrm{t}_{1}+\mathrm{t}_{2}>60 \mathrm{~s}$, drip), where "drip" means that the fused plastic droplet or plug is dropping from the burning sample.

\section{Morphological Observation and Atomic Mapping}

The cross section of the sample after burned was observed by using an electron beam 3D roughness analyzer, ERA-8800 (ELIONIX), at $10 \mathrm{kV}$. For the atomic mapping, an energy dispersive $\mathrm{X}$-ray analyzer, GENESIS 4000 SUTW (EDXA), was used. 


\section{Thermal Analysis}

Finely powdered test pieces prepared by crashing the samples at the liquid nitrogen temperature was used for the TG/DTA analyses by a TG/DTA6200 (Seiko Instruments) thermogravimetric/differential thermal analyzers under the following conditions: sample quantity $=5 \mathrm{mg}$, temperature range $=45$ $600^{\circ} \mathrm{C}$ at $10^{\circ} \mathrm{C} / \mathrm{min}$, under $\mathrm{N}_{2}$ atmosphere.

\section{Pyrolytic GC/MS}

The flame retardant powder $(0.01 \mathrm{~g})$ was thermally evaporated at $600^{\circ} \mathrm{C}$ by a double shot pyrolizer, PY-2020D (Frontier Laboratories), and the resulting gas was ionized by the electron impact method and analyzed by a GC/MS Automass Sun (JEOL) spectrometer. Mass number range of $20 \mathrm{~m} / z$ to $650 \mathrm{~m} / \mathrm{z}$ was recorded and characterized by comparing with the standard fragmentation mass spectrum patterns.

\section{RESULTS AND DISCUSSION}

According to the product information from the manufacturer, the addition of more than 20\% (by wt/wt throughout the paper) AFR or aluminum tris(diethylphosphinate) utilized in the present study is desirable to turn PA66 inflammable. However, the corresponding composite was difficult to fabricate reproducibly due to the powdery nature of AFR. Especially, if the corresponding composite was somehow managed to prepare, the brittleness or the lack of impact strength of the resulting products may not allow such a material to use in practical. Therefore, it is inevitable to decrease the quantity of AFR. The aim of the present paper is to enhance both the mechanical strength and the flame retardation properties of PA66 composites by using the dual flame retardants, AFR and RFR, in series. Before the examination of AFR/RFR/PA66 composites, the AFR/CR/PA66 composites were also evaluated.

In Table I the composites used in this paper were summarized. Every composite contained GF, Talc and $\mathrm{SiO}_{2}$ powder as reinforcement materials or inorganic fillers. As for the quantity and kind of flame retardation agent, the composites were classified into the following categories, i.e., Control: without AFR and RFR, I: with AFR and CR, II: with RFR, and $\boldsymbol{I I I -}$ $V$ : with AFR and RFR. In these composites, the total content of organic components, i.e., $\mathrm{AFR}+\mathrm{CR}+$ RFR+PA66 was kept constant to be 54\%. According to the $\gamma$-ray dose, the composite samples will be abbreviated as "Control-0" (Control sample with 0 kGy dose), "II-40" (composite II with 40 kGy dose), and so on.

The action of CR such as TAIC in PA66 or related thermoplastics to form network structures by $\gamma$-ray ir-

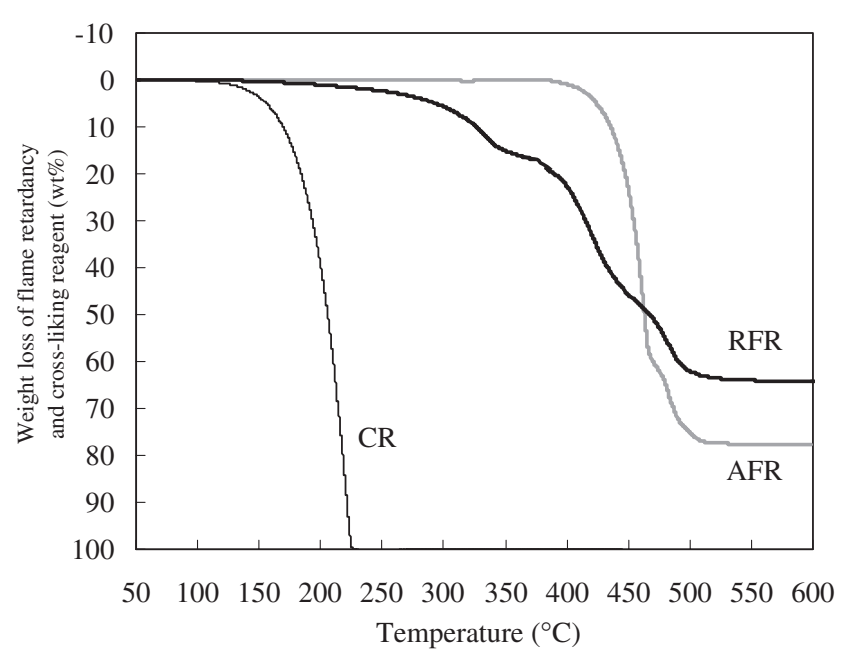

Figure 1. Thermal gravimetric analysis of CR, AFR and RFR.

radiation had been well-known..$^{8-11}$ As the creation of polyamide radical upon irradiation has been reported to occur at the methylene carbon adjacent to $>\mathrm{NH}$, the cross-linking reaction in the composite $I$ might proceed by the coupling reaction between ...-CO$\mathrm{NH}-{ }^{*} \mathrm{H}-\mathrm{CH}_{2}-\ldots$ and allyl radical of CR. ${ }^{12-17}$ From the structural similarity, we can expect the RFR molecules act as cross-linking reagent besides the flame retardant in the composites $\boldsymbol{I I I}-\boldsymbol{V}$, the mechanical strength of these composites was discussed at first.

\section{Temperature Dependence of Dynamic Viscoelasticity}

Figure 1 illustrates the TG curves of CR, AFR and RFR measured under $\mathrm{N}_{2}$ atmosphere. The kneading and extruding procedures to prepare the composites were carried out at $280^{\circ} \mathrm{C}$ where AFR obviously has enough stability in contrast to CR. Although the weight loss of a few $\%$ at $280^{\circ} \mathrm{C}$ was seen in RFR, it is not the serious problem in the course of kneading and extruding processes. The vaporization of RFR was apparently suppressed when mixed in PA66 matrix, probably because the RFR molecules have $\mathrm{P}=\mathrm{O}$ and $\mathrm{P}-\mathrm{N}$ functionalities that may interact with the N-H groups of fused PA66 through the hydrogen-bonding.

In Figures 2(a) and (b), the temperature dependence curves of storage modulus $\left(G^{\prime}\right)$ and loss tangent $(\tan \delta)$ for the Control-0 and -40 composite samples were illustrated. The drastic decrease of $G^{\prime}$ in accordance with the appearance of $\tan \delta$ peak was observed at about $240-250^{\circ} \mathrm{C}$, where the Control samples began to deform by fusion. As there were little differences between the values of Control-0 and - 40 samples, the cross-linking did not progress upon irradiation in the Control samples that contained no CR and RFR.

Because AFR is the powdery solid, the composite 

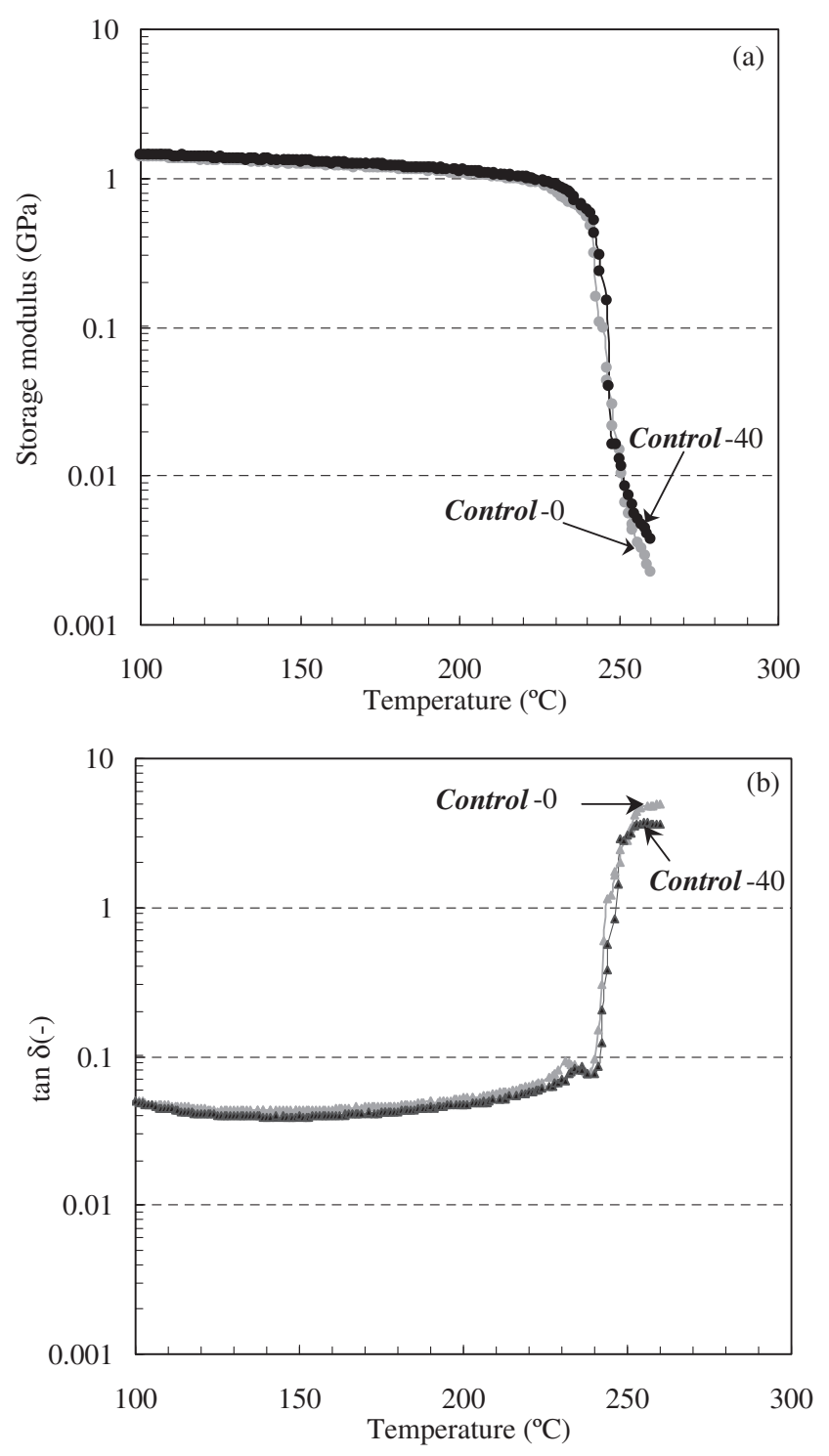

Figure 2. Temperature dependence of (a) $G^{\prime}$ and (b) $\tan \delta$ in Control-0 and -40 .

containing $12 \%$ (or more) AFR as well as inorganic fillers was brittle at r.t. and was softened beyond the fusion temperature, and in total, did not give satisfactory mechanical strength. However, the co-existence of $2 \%$ CR besides $12 \%$ AFR and the subsequent $\gamma$ ray irradiation have changed the composite nature endurable to mechanical disturbance and heating. The temperature dependence curves of $G^{\prime}$ and $\tan \delta$ for $\boldsymbol{I}$ before and after the $\gamma$-ray irradiation were compared in Figures 3(a) and (b), respectively. As for $I-0$, it began to fuse at the temperature higher than $240{ }^{\circ} \mathrm{C}$ to give the drastic decrease of $G^{\prime}$ with the appearance of $\tan \delta$ peak. In accordance with the progress of crosslinking or with increasing the $\gamma$-ray dose, the $G^{\prime}$ values of $\boldsymbol{I}-25$ and $\boldsymbol{I}-40$ shown in Figure 2(a) did not drop so steeply as in the cases of Control-0/-40 and I-0 samples, while the $\tan \delta$ peak in the $240-260^{\circ} \mathrm{C}$ range of $\boldsymbol{I}-25$ and $\boldsymbol{I}-40$ in Figure 3(b) became very small.
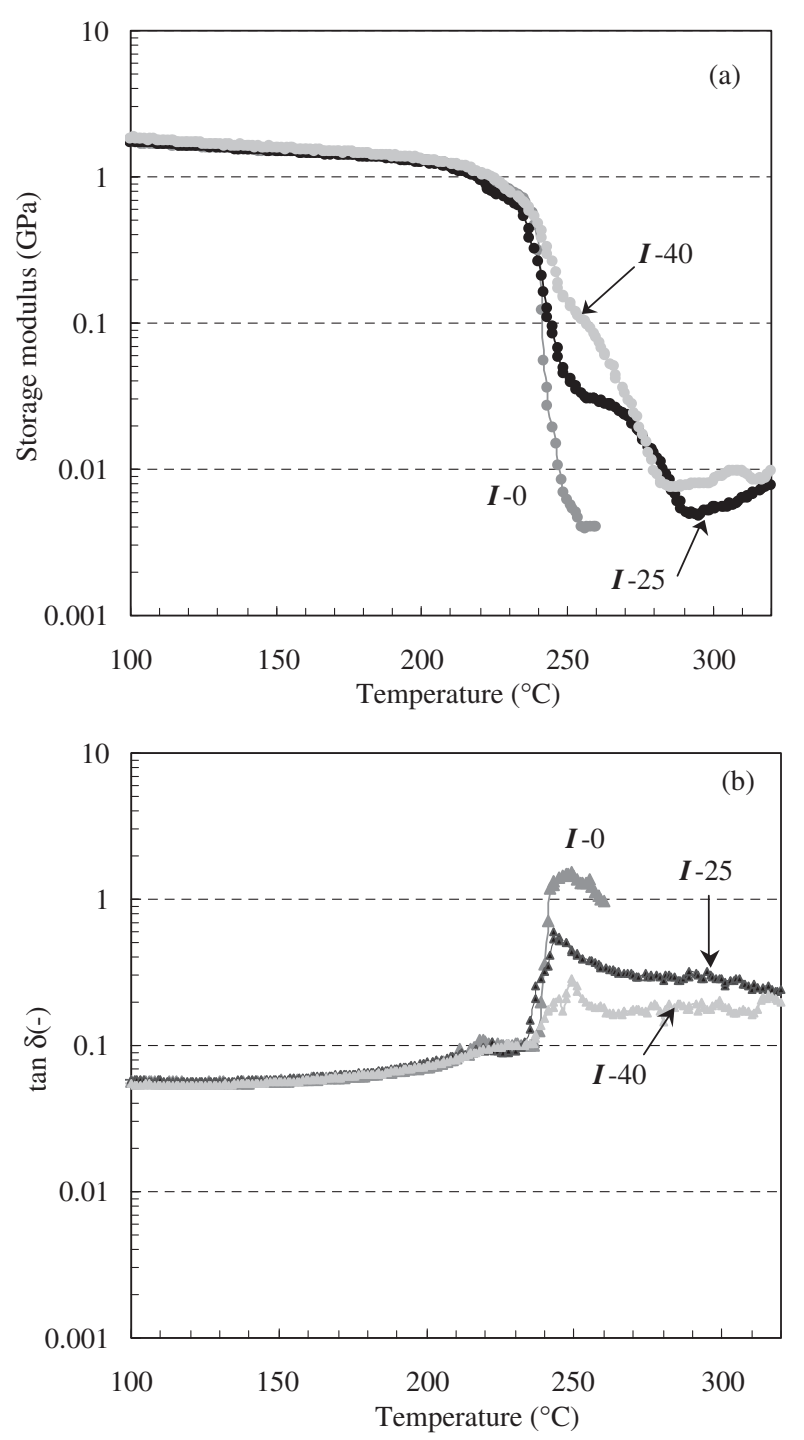

Figure 3. Temperature dependence of (a) $G^{\prime}$ and (b) $\tan \delta$ in I-0, -25 and -40 .

When the $G^{\prime}$ value at $250{ }^{\circ} \mathrm{C}$ was chosen for example, the $25 \mathrm{kGy}$ dose was enough to give the mechanical strength of $0.03 \mathrm{GPa}$ satisfactory to the conventional use as housing and casing material of electric equipments. At more elevated temperature beyond the fusion temperature of uncross-linked PA66 such as $285-310^{\circ} \mathrm{C}$, the $G^{\prime}$ value of $\boldsymbol{I}-25$ and $\boldsymbol{I}-40$ was recovering to larger value with the temperature elevation showing the rubbery elasticity and non-fusible nature due to network formation. ${ }^{17}$

The results of similar attempts for $\boldsymbol{I I}$ before and after the $\gamma$-ray irradiation containing 12\% RFR were illustrated in Figures 4(a) and (b). Although the $G^{\prime}$ curve of $\boldsymbol{I I}-\mathbf{0}$ was very similar to that of the Control or I-0 samples, the decrease of $G^{\prime}$ value of $\boldsymbol{I I}-25$ and $\boldsymbol{I I}-40$ at the elevated temperature range was very much suppressed, and it still kept $0.3 \mathrm{GPa}$ or more at $250^{\circ} \mathrm{C}$ being 10 times larger than the value described above for 

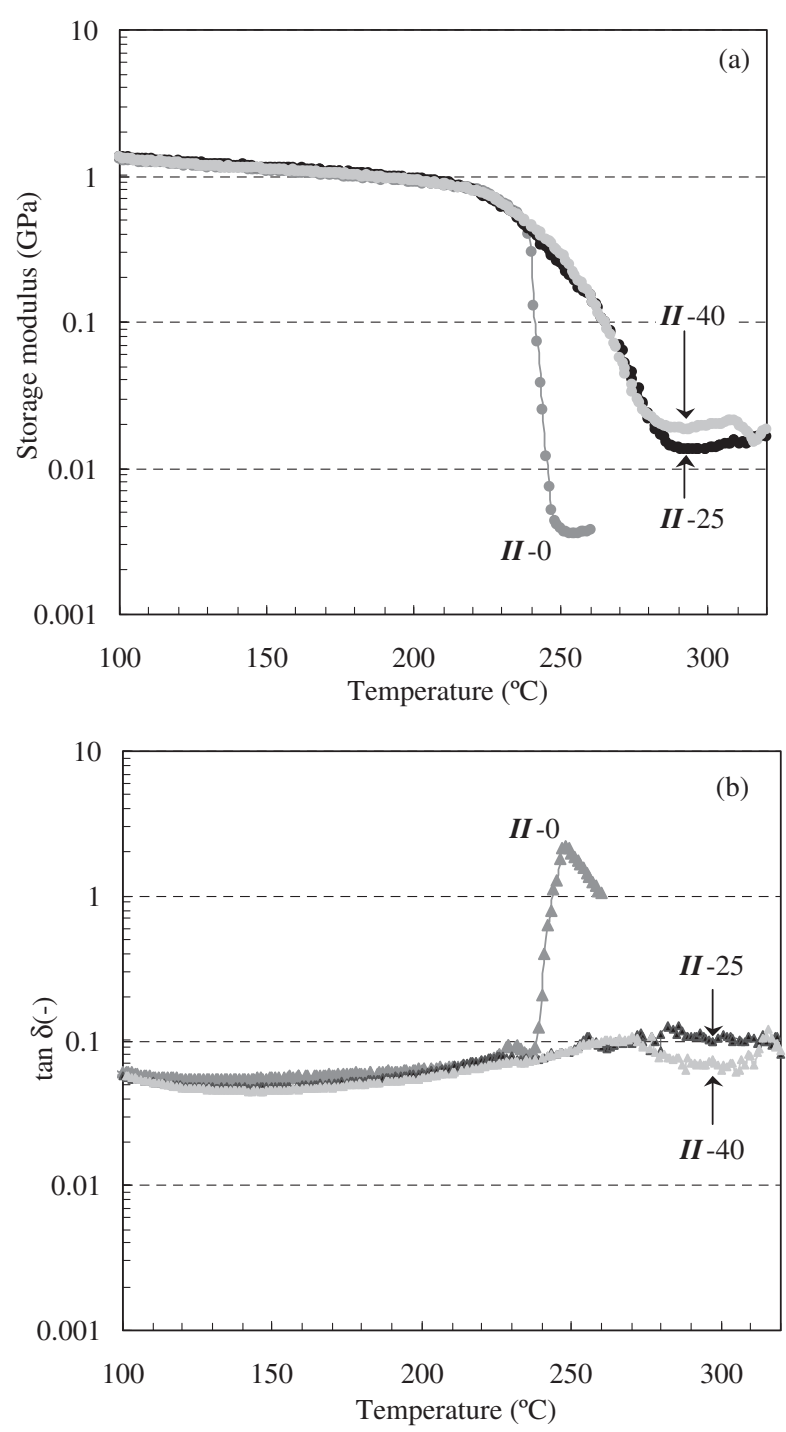

Figure 4. Temperature dependence of (a) $G^{\prime}$ and (b) $\tan \delta$ in II-0, -25 and -40 .

$\boldsymbol{I}-25$ and $\boldsymbol{I}-40$. As for the temperature dependence profiles of $\tan \delta$, unlike the change from $\boldsymbol{I}-0$ to $\boldsymbol{I}-25$ or $\boldsymbol{I}$ 40 , the $\tan \delta$ peak at about $240{ }^{\circ} \mathrm{C}$ seen in $I I-0$ completely disappeared in $\boldsymbol{I I}-25$ and $\boldsymbol{I I}-40$. These results might be attributed to that the degree of cross-linking of $\boldsymbol{I I}$ is considered to be higher than that of $\boldsymbol{I}$, when compared at the same $\gamma$-ray dose, due to the increased concentration of allyl functional groups in II. At more elevated temperature, such as $285-310^{\circ} \mathrm{C}$, the recovery of $G^{\prime}$ value was again noticed in $\boldsymbol{I I}-25$ and $\boldsymbol{I I}-40$ as like $\boldsymbol{I}-25$ and $\boldsymbol{I}-40$ showing the rubbery elasticity and non-fusible nature due to network structure. ${ }^{17}$ Comparing the $G^{\prime}$ and $\tan \delta$ values in Figures 3 and 4, difference between $\boldsymbol{I}-25$ and $\boldsymbol{I}-40$ or difference between $\boldsymbol{I I}-25$ and $\boldsymbol{I I}-40$, respectively, was relatively small and the effect of $\gamma$-ray irradiation cross-linking seemed to saturate at the $40 \mathrm{kGy}$ dose. Note that this assumption was not directly related to the complete reaction of every allyl group. When the network for-
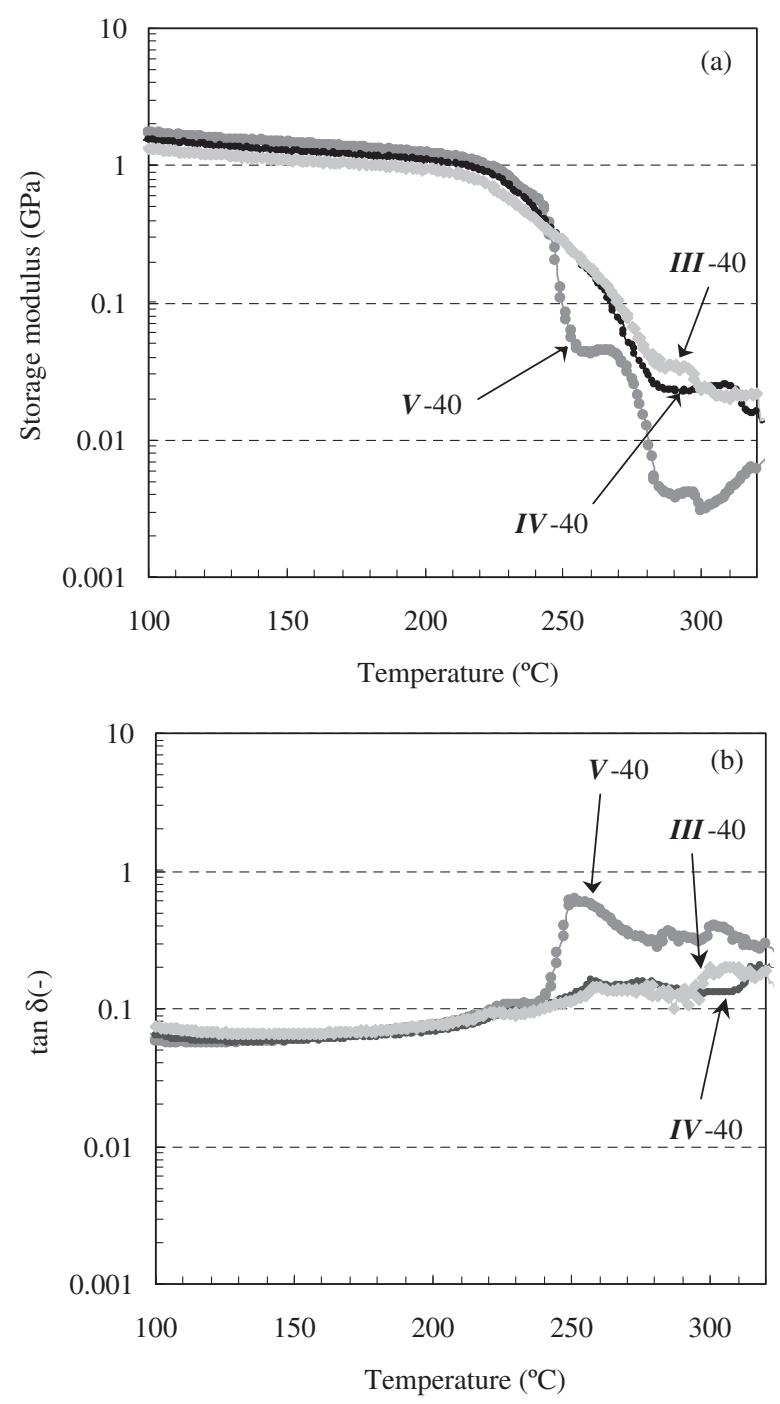

Figure 5. Temperature dependence of (a) $G^{\prime}$ and (b) $\tan \delta$ in III-40, $I V$-40 and $\boldsymbol{V}$-40.

mation was commenced to certain degree, further cross-linking might not progress due to the decreased freedom of RFR group motion and therefore, some amount of allyl groups were remained unreacted.

The AFR+RFR mixed composites, i.e., the $\boldsymbol{I I I}-\boldsymbol{V}$ composites were examined next. Unfortunately, the $12 \%$ AFR $+12 \%$ RFR mixed composite was difficult to prepare reproducibly due to too low PA66 quantity, the content of RFR was changed to 8,4 and $2 \%$ for the III- $\boldsymbol{V}$ composites, respectively. In Figures 5(a) and (b) the temperature dependence curves of $G^{\prime}$ and $\tan \delta$ in $\boldsymbol{I I I}-40, \boldsymbol{I V}$-40 and $\boldsymbol{V}$-40 were illustrated. While the $G^{\prime}$ and $\tan \delta$ profiles of $\boldsymbol{I I I}-40$ and $\boldsymbol{I V}$-40 at the temperature range of $240{ }^{\circ} \mathrm{C}$ or more were very similar to $\boldsymbol{I I}-25$ or $\boldsymbol{I I}-40$ shown in Figures 4(a) and (b), those of $\boldsymbol{V}$-40 revealed that the cross-linking in $\boldsymbol{V}$-40 is somewhat more loose than in III-40 and $I V$-40.

Since RFR is a kind of cross-linking reagent with 8 allyl groups per a molecule, it is interesting to com- 


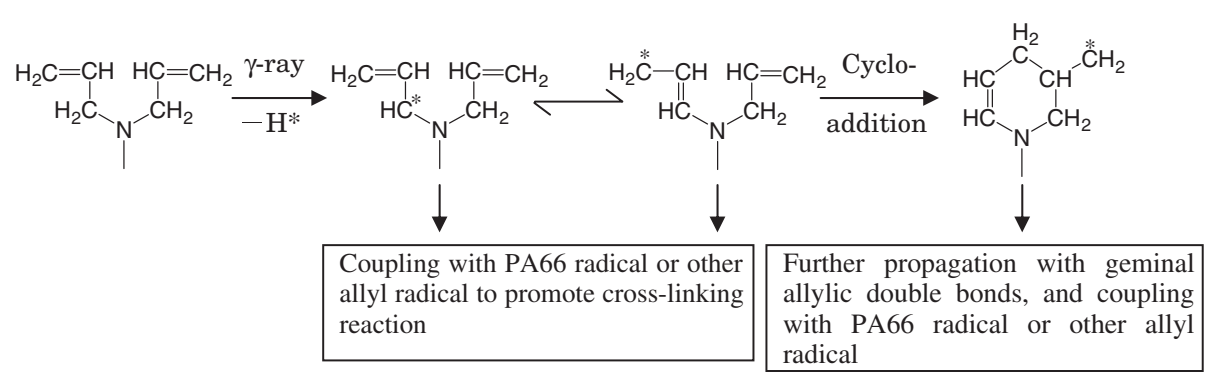

Scheme 2.

pare the composites $\boldsymbol{I}$ and $\boldsymbol{V}$ containing $2 \% \mathrm{CR}$ and $2 \%$ RFR, respectively. The $G^{\prime}$ and $\tan \delta$ profiles of $\boldsymbol{V}$-40 were very similar to those of $\boldsymbol{I}-25$. As for the reactivity of geminal diallyl groups such as the diallylamino-functionality of the present RFR molecule, the intramolecular cycloaddition process has been known in the field of polymer synthesis as follows: ${ }^{18}$

In general, the stabilized resonance structure of allyl radicals (center 2 formulae in Scheme 2) does not allow the radical propagation to advance the addition polymerization of carbon-carbon double bonds. Therefore, these allyl radicals might be the major species to promote cross-linking with PA66 radicals by coupling reaction. On the other hand, the occurrence of cycloaddition reaction to yield an ordinary primary carbon radical (right-most formula in Scheme 2) decreased the degree of cross-linking, not only because the functionality per a RFR molecule possibly decreased from 8 to 4 but also the primary carbon radical was able to promote further cycloaddition polymerization of geminal diallyl groups. The cycloaddition reaction would also increase the local segmental motion of PA66 strands due to the creation of free volume by the insertion of bulky 6-membered ring structures among the strands. Thus in total, it is reasonable that the network formation in $\boldsymbol{V}$-40 was rather loose in comparing with $\boldsymbol{I}-40$, but still good enough to became non-fusible and to maintain the $G^{\prime}$ value of more than $0.03 \mathrm{GPa}$ at $250^{\circ} \mathrm{C}$ that is the minimal requirement to endure the soldering process as for the housing materials of electric equipments.

\section{Flame Retardation}

The grade of UL94/V test and the combustion characteristics of the composites were also shown in Table I. In order to evaluate the char formation, the composite samples were conducted to TG analyses, and the typical examples for $40 \mathrm{kGy}$-irradiated samples were illustrated in Figure 6. Because the composite samples contained non-volatile fillers and inorganics, the vertical axis of Figure 6 was standardized in respect to the amount of organics. The aluminum atoms of AFR were regarded as organics, since it was difficult to know in what structure the aluminum

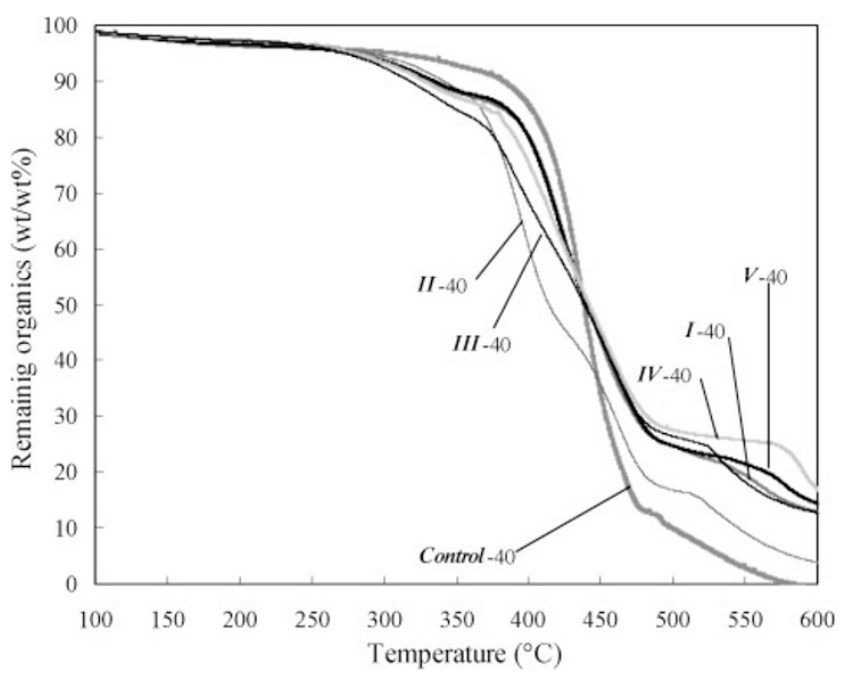

Figure 6. Thermal gravimetric analysis of Control-40, I-40, II-40, III-40, IV-40 and $\boldsymbol{V}$-40. See text as for the explanation of vertical axis.

atoms existed after the thermal degradation of AFR. At any rate, it gave only small difference to the vertical axis calculation that whether the aluminum portion was taken into account or not. The char yield was determined to be the percentage of remaining organics at $600{ }^{\circ} \mathrm{C}$ in respect to the total organics, i.e., remaining organics $(\mathrm{wt} / \mathrm{wt} \%)=100 \times[($ sample weight after heating - weight of inorganics)/initial weight of total organics].

Control-0 or -40 as well as pure PA66 showed drip-burn (see Experimental) upon combustion, corresponding to "NG" in the UL94/V flammability examination. Note that almost no char was found in these samples. On the other hand, in the case of $\boldsymbol{I}$ and $\boldsymbol{I I I}-\boldsymbol{V}$ composites with UL94/V-1 or better grade, even the uncross-linked samples gave no dripping, as the flare was spontaneously fading within at least $30 \mathrm{~s}$ and more than $10 \%$ of char was formed by the action of AFR. The composites II-0-II-40 with UL94/NG grade burned continuingly for about $75 \mathrm{~s}$, but without dripping. Though the reason of no drippings was unclear, it is considered that relatively high char yield of 6-7\% or possible cross-linking via RFR functionality at elevated temperature during burning helped to 


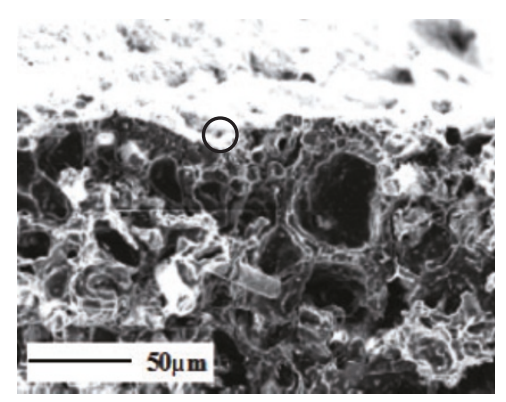

(a)

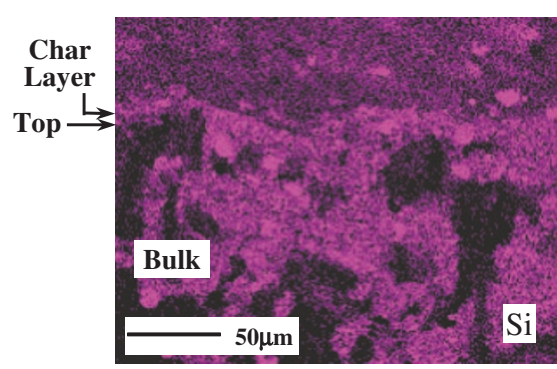

(c)

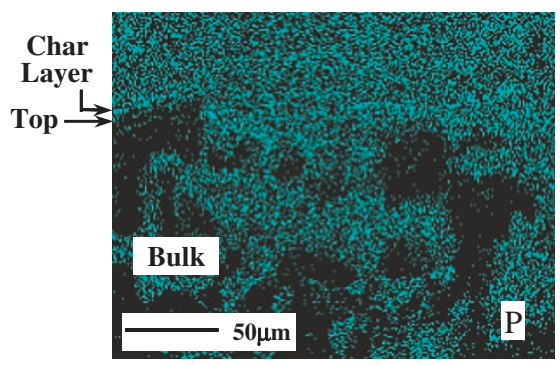

(e)

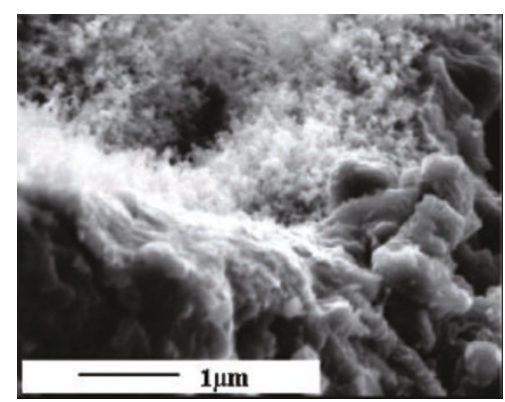

(b)

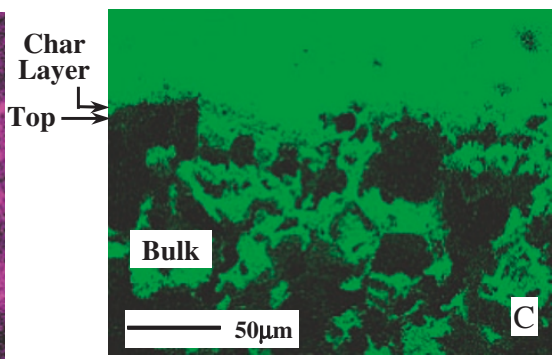

(d)

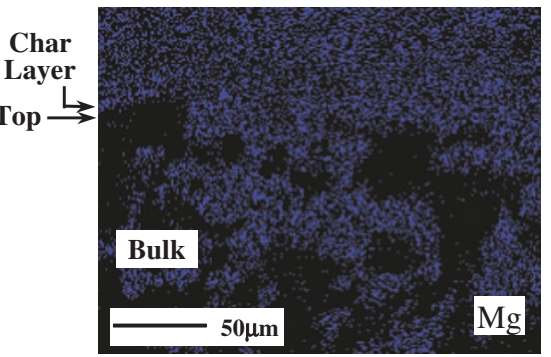

(f)

Figure 7. Continued on next page.

maintain the sample shape.

Although the composite II containing solely RFR did not give notable flame retarding effect, the composites $\boldsymbol{I}$ and $\boldsymbol{I I I}-\boldsymbol{V}$ containing $\mathrm{AFR}(+\mathrm{CR})$ or AFR + RFR showed the flame retarding effect better than UL94/V-1 grade. Especially, the composite $\boldsymbol{V}$ gave the best result, UL94/V-0 grade. Approximate burning period, $t_{1}$ or $t_{2}$ (see Experimental), was as follows, $\boldsymbol{I}: 25, \boldsymbol{I I}: 75-90, \boldsymbol{I I I}: 20, \boldsymbol{I V}: 10-15, \boldsymbol{V}: 5-9$ s, respectively. In general, the trapping and inactivation of the combustive fragments by phosphorus radicals were believed to be the initial and major events of the flame retardation followed by the formation of carbonaceous (char) layers that prevent the further heat flux and fresh oxygen income. ${ }^{19,20}$ However, the flame retardation properties fluctuated in-between of UL94/V-1 and $\mathrm{V}-0$ as described above in the composites $\boldsymbol{I}$ and $\boldsymbol{I I I}-\boldsymbol{V}$ nevertheless gave very similar char yield of 10-13\%. Such difference in flame retardation effect was considered to be due to the behavior of AFR and/or RFR fragments in fused resins. Therefore, the char formation effects of AFR and RFR as well as the characteristics of resulting chars were studied about the $\boldsymbol{I I}-40$ and $\boldsymbol{V}$-40 samples by SEM and EDX (energy dispersive $\mathrm{X}$-ray analysis).

Because the results of UL94/V test for II-40 was NG, the samples used for SEM/EDX observation were prepared by intentional extinguishing after burnt for $10 \mathrm{~s}$. In the case of $\boldsymbol{V}-40$, the samples after the UL94/V test were directly used for the observation. In Figure 7(a), the SEM cross-sectional view of burnt II-40 was shown, in which the char layer formation was very difficult to notice. From the more magnified image shown in Figure 7(b), the surface was revealed to be covered with thin and fluffy char layers. Judging from the corresponding elemental mapping EDX images in Figures 7(c-f), silicone (originating to glass fiber \& $\mathrm{SiO}_{2}$ gel powder), phosphorus, carbon and magnesium (originating to Talc) atoms were homogeneously distributed from the surface to the deep positions except for the void spaces. No distinct localization of phosphorus and carbon atoms to the fluffy char layers was occurred. These results indicated that the migration of phosphorus-containing fragments to the 


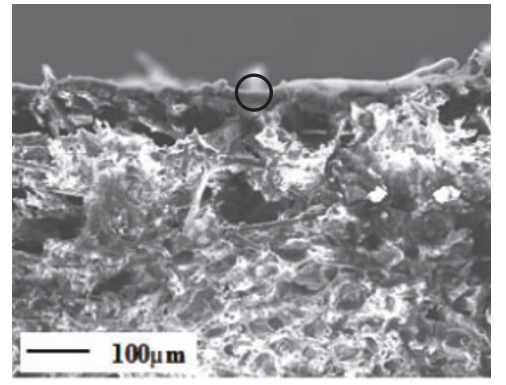

(g)

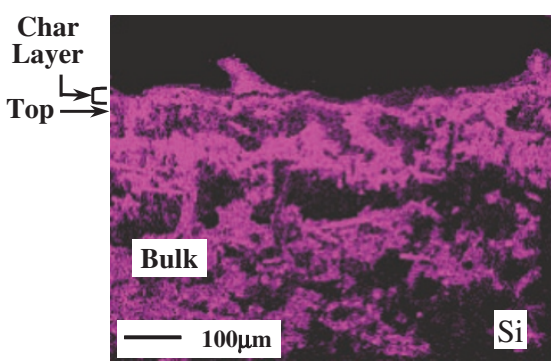

(i)

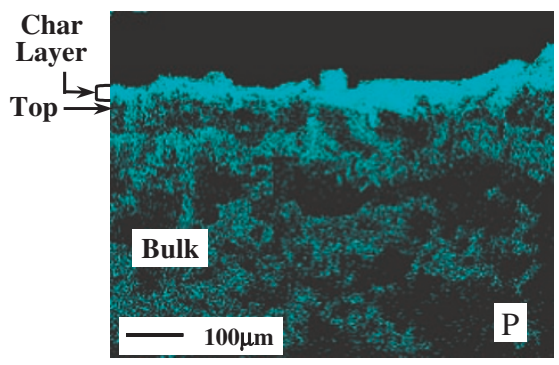

(k)

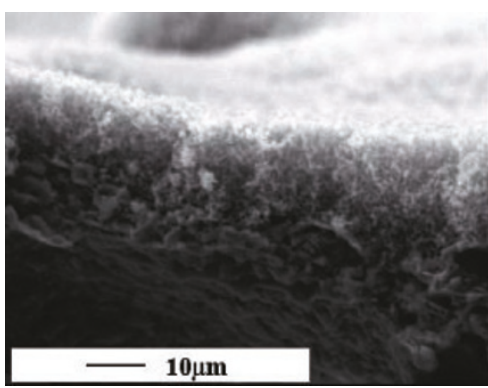

(h)

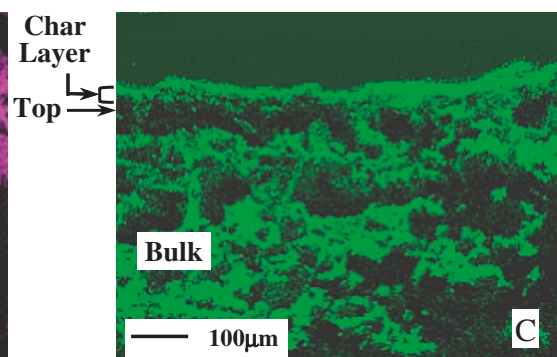

(j)

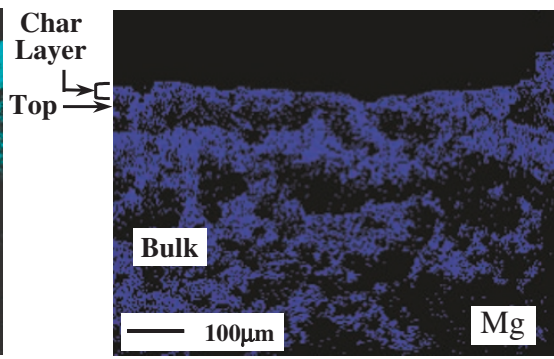

(1)

Figure 7. SEM-EDX micrographs of $\boldsymbol{I I}-40$ and $\boldsymbol{V}$-40 crosslinked and burnt. (a)-(f): $\boldsymbol{I I}-40,(\mathrm{~g})-(\mathrm{l}): \boldsymbol{V}-40$, (a, g): SEM cross-sectional view, (b, h): SEM local surface image, (c, i): Si-, (d, j): C-, (e, k): P- and (f, l): Mg-mapping by EDX, respectively.

surface was insufficient in $\boldsymbol{I I}-40$ to commence the rigid char layer formation during the combustion. Same observations were accomplished for $\boldsymbol{V}-40$. The SEM cross-sectional view and magnified surface image of $\boldsymbol{V}$-40 after the flammability test were shown in Figures $7(\mathrm{~g})$ and $(\mathrm{h})$, respectively. One can distinguish the surface of burnt $\boldsymbol{V}$-40 sample was covered with the dense char layers. Note that the char layers were created at the outside of the original composite surface. Further comparison between the SEM image and the EDX images [Figures 7(i-l)] indicated that the phosphorus atoms were concentrated in the char layers. Because the homogeneous distribution of immobile silicone and magnesium atoms was maintained even after the combustion, we can imagine that the phosphorus-containing fragments migrated through the resin bulk to reach the gas phase and the consequently occurring imperfect combustion produced the char layers overlain the composite surface of $\boldsymbol{V}-40$.

By utilizing the distribution of immobile $\mathrm{Mg}$ atoms as standard, the $[\mathrm{P}] /[\mathrm{Mg}]$ ratio of $\boldsymbol{I I}-40$ and $\boldsymbol{V}-40$ be- fore and after the combustion at three different positions, top, middle and center, gave interesting results as shown in Figures 8(a) and (b), where three positions are designated as follows, top: the surface position of composites, just beneath the char layers in the case of burnt sample, middle: about $0.2 \mathrm{~mm}$-depth position from the top, center: about $0.4 \mathrm{~mm}$-depth position from the top corresponding to a halfway of total thickness of $0.8 \mathrm{~mm}$. Unfortunately the $[\mathrm{P}] /[\mathrm{Mg}]$ ratio at the char layers could not be determined, because the reproducible EDX observation pin-pointedly focused at the narrow char layer bands was difficult due to the rough and uneven cross-sectional surface of char layer bands. Before burnt, the ratio was constant at every position within the limit of error. After burnt, the ratio in $\boldsymbol{I I}-40$ became a half of the initial value at every position. On the other hand, the ratio in $\boldsymbol{V}$-40 after the combustion changed according to the position, such as, @top: 3-4-times larger than the initial value, @middle: decreased to almost 0 , @center: a half of the initial value. From the P-mapping EDX image 


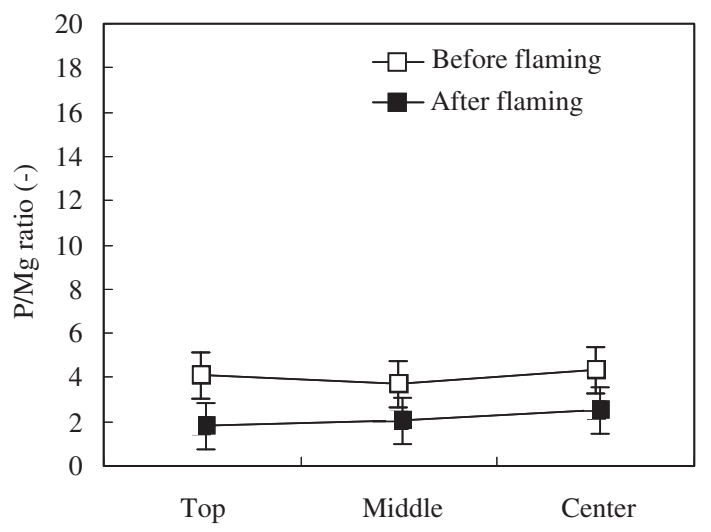

(a)

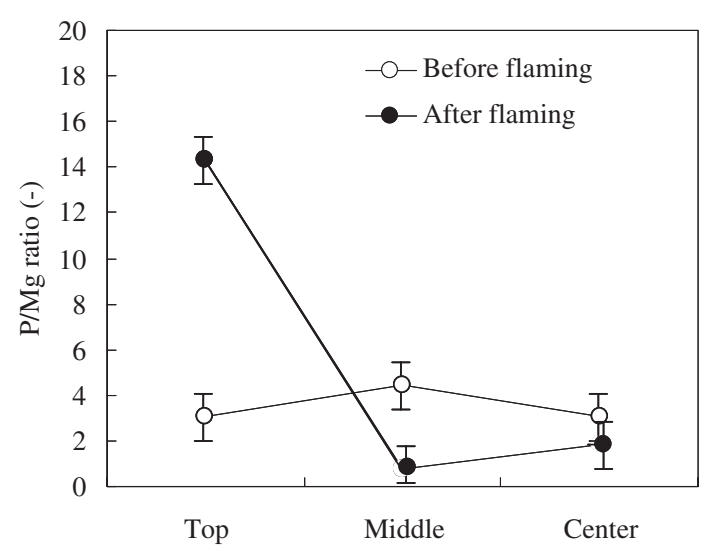

(b)

Figure 8. $[\mathrm{P}] /[\mathrm{Mg}]$ ratio at different positions of (a) $\boldsymbol{I I}-40$ and (b) $\boldsymbol{V}-40$ before and after burnt. top: the surface position of composites, just beneath the char layers in the case of burnt sample, middle: about $0.2 \mathrm{~mm}$-depth position from the top, center: about $0.4 \mathrm{~mm}$-depth position from the top, corresponding to a halfway of total thickness, $0.8 \mathrm{~mm}$.

photograph of Figure 7(k) for the burnt $\boldsymbol{V}$-40 sample, the phosphorus concentration at the char layers was apparently very much higher than that at top. Therefore, the phosphorus concentration in $V$-40 was in the order, @charlayers $\gg @ t o p>@ m i d d l e(=0)$.

These results indicated that steep evolution of phosphorus-containing fragments was derived mainly from AFR and was necessary to create dense char layers. However, this explanation is not good enough to understand the difference of UL94/V grade among the composites $\boldsymbol{I}$ and $\boldsymbol{I I I}-\boldsymbol{V}$, as these composites contained the same amount of AFR, 12\%. Thus we should consider about the role of RFR on the flame retardation besides the network formation.

In order to know the radical trapping effect, the fragmentation properties of AFR and RFR were studied by the pyrolytic GC/MS spectroscopy. From the TG curves of AFR and RFR illustrated in Figure 1, both flame retardants were completely decomposed at the temperature higher than $500^{\circ} \mathrm{C}$. In Table II were summarized the results of pyrolytic GC/MS spectroscopy at $600^{\circ} \mathrm{C}$. As imagined from the steep weight-loss profile of the TG curve of Figure 1, AFR gave a single peak spectrum that revealed the evolution of the specific fragment containing two phosphorus atoms with $M_{\mathrm{w}}$ of about 198 [Table II(a)], very close to $M_{\mathrm{w}}$ of plausible degradation product of $\mathrm{AFR}$, i.e., $\left[\left(\mathrm{C}_{2} \mathrm{H}_{5}\right)_{2} \mathrm{P}\right]_{2} \mathrm{O}\left(M_{\mathrm{w}}=194\right), \mathrm{C}_{2} \mathrm{H}_{5} \mathrm{P}(=\mathrm{O})-$ $\mathrm{O}-\mathrm{P}(=\mathrm{O})\left(\mathrm{C}_{2} \mathrm{H}_{5}\right)_{2}\left(M_{\mathrm{w}}=197\right)$, and so on. At any rate, it was clarified that AFR steeply evolved rather low molecular weight diphosphorus fragment at 450$500^{\circ} \mathrm{C}$. On the other hand, the multi-step degradation of RFR as seen in gradual weight-loss profile of Figure 1 formed various fragments of which several species were summarized in Table II(b), and the corresponding total ion chromatograms (TICs) of evolved gas at $600^{\circ} \mathrm{C}$ were illustrated in Figure 9. Pyrrole de-
Table II. Fragment species of flame retardant degradation at $600{ }^{\circ} \mathrm{C}$

(a) AFR

\begin{tabular}{ccc}
\hline Peak no.(\#) & Compound & $M_{\mathrm{w}}$ \\
\hline $1^{\mathrm{a}}$ & Diphosphorus compound & 198 \\
\hline
\end{tabular}

${ }^{\mathrm{a}}$ Estimate.

(b) RFR

\begin{tabular}{ccr}
\hline Peak no.(\#) & Compound & $M_{\mathrm{w}}$ \\
\hline 1 & Propene & 42 \\
2 & Pyrrole & 67 \\
3 & 3-Metylpyrrole & 81 \\
4 & 4-Phenylphenol & 170 \\
5 & {$\left[1,1^{\prime}\right.$-Biphenyl $]-4,4^{\prime}$-diol } & 186 \\
$6^{\mathrm{b}}$ & Phosphorus compound & 424 \\
\hline
\end{tabular}

${ }^{\mathrm{b}}$ Estimate.

rivatives [peak \#2,3 in Table II(b)] formed by the cyclization and dehydrogenation of diallylamine groups were the typical low molecular weight fragments as well as propene $\left(M_{\mathrm{w}}=42\right.$, peak \#1), allyl radical $\left(M_{\mathrm{w}}=41\right.$, peak \#1 ?) or other low molecular weight hydrocarbons. Besides the biphenyl derivatives (peak $\# 4,5$ ) corresponding to the corpus of RFR, the evolution of $\left[\left(\mathrm{CH}_{2}=\mathrm{CHCH}_{2}\right)_{2} \mathrm{~N}\right]_{2} \mathrm{P}(=\mathrm{O}) \mathrm{O}-\mathrm{Ph}-\mathrm{Ph}-{ }^{*}\left(M_{\mathrm{w}}=\right.$ 424 , peak \#6) formed by the elimination of one bis(diallylamino)phosphoryl group was seen. As far as the big fragment, \#6, was evolved, it is reasonable to consider that other phosphorus-containing fragments such as $\left[\left(\mathrm{CH}_{2}=\mathrm{CHCH}_{2}\right)_{2} \mathrm{~N}\right]_{2} \stackrel{*}{\mathrm{P}}(=\mathrm{O})$ or related species were formed during the combustion. Unfortunately, the existence of too many small peaks in such molecular weight region did not allow further detailed assignment.

From these results, the authors would like to spec- 


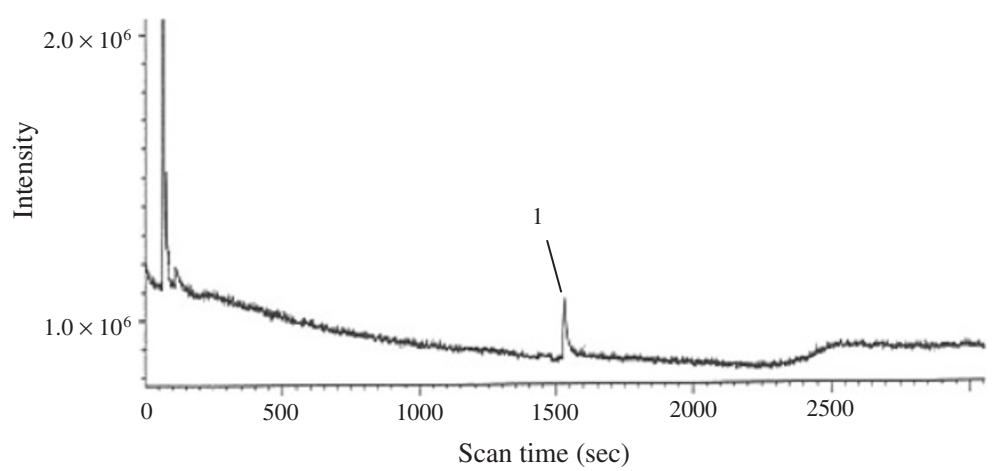

(a)

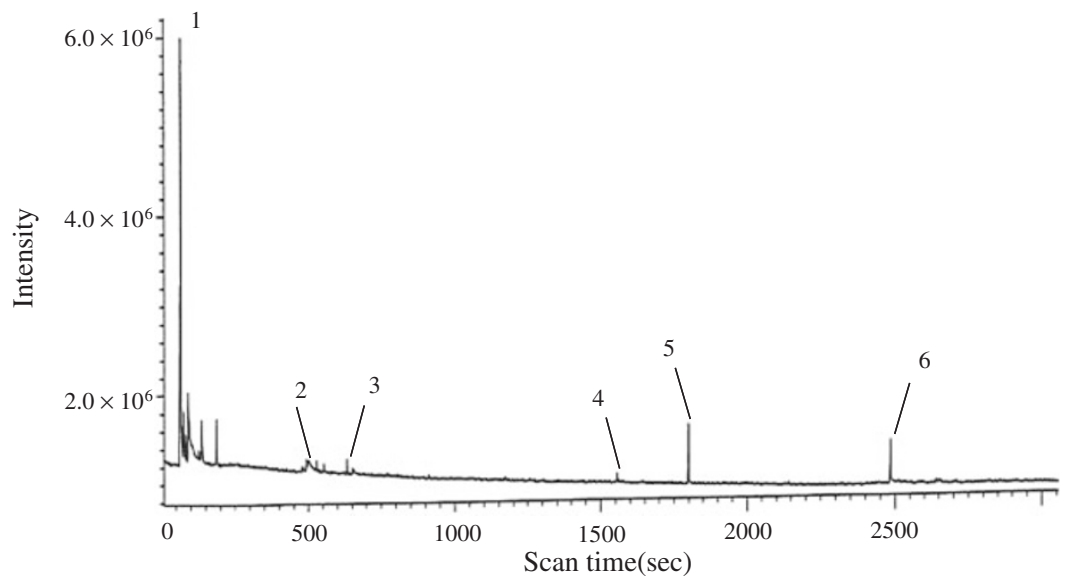

(b)

Figure 9. Total ion chromatogram of GC-MS, (a) AFR, (b) RFR.

ulate that the reason of UL94/V-0 in the composite $\boldsymbol{V}$ was due to the concerted effect of AFR and RFR fragments. The steep evolution of rapidly migrating diphosphorus-containing low molecular weight fragments from AFR might be the major source to form the char layers but might not be sufficient to give enough rigidity to the char layers. The larger RFR fragments carrying phosphorus and/or nitrogen atoms and functional groups were thought to enhance the nucleation for the AFR fragment recombination and therefore the char layers formed in $\boldsymbol{V}$ were satisfactory rigid to show UL94/V-0 grade.

In order to realize such concerted effect, the AFR and RFR contents should be precisely balanced, because the composites with RFR $\geq 4 \%(\boldsymbol{I I I}, \boldsymbol{I V})$ gave the lesser flame retardation grade. As described above, RFR evolved combustive gases such as propene besides the larger fragments, higher content of RFR possibly decreased the flame retardation grade.

When the samples of same composite but with different $\gamma$-ray dose were compared, smaller $\gamma$-ray dose gave shorter burning period of UL94/V test. In every case the burning period of Composite-0 was shorter than that of Composite-40, probably because the fragment migration through the composite bulk became some extent more difficult by the network formation.
But the difference in the burning period of Composite -0 and Composite- 40 was merely a few seconds in every case, it is noteworthy that the mechanical strength of composites was evidently improved by the cross-linking without decreasing significantly the flame retarding properties.

\section{CONCLUSIONS}

By the AFR-RFR dual incorporation and subsequent $\gamma$-ray irradiation cross-linking, the PA66 composites with the flame retardation properties of UL94/ $\mathrm{V}-1-\mathrm{V}-0$ grade as well as satisfactory mechanical strength at the temperature where the ordinary PA66 resin fused were established. RFR was considered to play an important role not only on the network formation but also on the improvement of flame retardation properties. Steep evolution of phosphorus-containing fragments was necessary for the dense char layer formation that was characteristic in the AFR-RFR dual composites with UL94/V-0 grade.

Acknowledgment. This research was partially supported by the COE Strategic Research Initiative Program for Future Nano-science and Technology of Tokyo Univ. of Agricul. \& Technol. 


\section{REFERENCES}

1. "Flame Retarder Usage Manual," H. Nishizawa, Ed., Technonet, Matsudo, 2002, p 320.

2. Y. Miwa, H. Ishida, T. Kanno, H. Yanase, and K. Shigehara, Kobunshi Ronbunshu, 63, 799 (2006).

3. G. Camino, L. Costa, and G. Martinasso, Polym. Degrad. Stab., 23, 359 (1989).

4. S. Jahromi, W. Gabriëlse, and A. Braam, Polymer, 44, 25 (2003).

5. E. Jenewein, H.-J. Kleiner, W. Wanzke, and W. Budzinsky, (to Clariant), US Patent 6,365,071 (2002).

6. E. Schlosser, B. Nass, and W. Wanzke, (to Clariant), US Patent 6,503,969 (2001).

7. S. V. Levchik and E. D. Well, J. Fire Sci., 24, 345 (2006).

8. H. Liang and W. Shi, Polym. Degrad. Stab., 84, 525 (2004).

9. A. Matsumoto, Prog. Polym. Sci., 26, 189 (2001).

10. A. Charlesby and D. Campbell, "ESR Application to
Polymer Research,” John Willy and Sons, New York, 1973, p 147.

11. DW. Lee, Kunststoffe, 83, 127 (1993).

12. R. Sengupta, V. K. Tikku, A. K. Somani, T. K. Chaki, and A. K. Bhowmick, Radiat. Phys. Chem., 72, 625 (2005).

13. J. Zimmerman, J. Polym. Sci., 46, 151 (1960).

14. N. Vasanthan, N. Murthy, and R. Bray, Macromolecules, 31, 8433 (1998).

15. M. Igarashi, J. Polym. Sci., Polym. Chem. Ed., 21, 2405 (1983).

16. C. Birkinshaw, M. Buggy, and S. Daly, Mater. Chem. Phys., 17, 239 (1987).

17. C. Deeley, A. Woodward, and J. Sauer, J. Appl. Phys., 28, 1124 (1957).

18. G. B. Butler and R. J. Angelo, J. Am. Chem. Soc., 79, 3128 (1957).

19. S. V. Levchik and E. D. Weil, Polym. Int., 49, 1033 (2000).

20. Y. Chang, Y. Wang, D. Ban, B. Yang, and G. Zhao, Macromol. Mater. Eng., 289, 703 (2004). 